\title{
Hacia Una Política De Salud Integrada: las LICENCIAS OBLIGATORIAS Y LA PRODUCCIÓN PÚBLICA DE MEDICAMENTOS
}

\author{
Marta Carolina Giménez Pereira* \\ Salete Oro Boff **
}

\begin{abstract}
1 Introducción. 2 Licencias obligatorias. 3 El caso de las licencias obligatorias en el campo de la salud. La experiencia internacional y la Propia Mexicana. 4 La relevancia de la noción de salud dentro de las políticas públicas: la producción pública de medicamentos (PPM) y redefinición del concepto de burocracia en el sistema de salud. 5 La innovación terapéutica, la investigación médica con la contribución del paciente y las unidades productoras de medicamentos (UPM). 6 Principales principios activos elaborados por laboratorios públicos en Argentina. 7 Conclusiones. Referências.
\end{abstract}

\section{RESUMEN}

El objetivo del trabajo es describir las Licencias Obligatorias y presentar los impactos producidos en la flexibilización de la protección de las patentes farmacéuticas al reconocer un derecho a medidas alternativas a favor de la salud pública y de la promoción del acceso a las medicinas. Además, introduce la noción de Producción Pública de Medicamentos por el sector público como instrumento de política pública y que trata puntos estratégicos del sector salud, intentando reforzarlo con respeto a la propiedad intelectual, estimulando la I $+\mathrm{D}$, conectando sector público y privado, garantizando abastecimiento y distribución y resultando el reforzamiento de la dinámica de innovación nacional. Método: comparativo y deductivo y la investigación bibliográfica.

Palabras-clave: Producción pública de medicamentos. Innovación tecnológica. Propiedad intelectual. Patentes farmacéuticas. Licencias obligatorias o compulsivas.

* Alumna de post-doctorado junto a UNAM - México, PPGD Capes - IMED. Doctora en Derecho / Instituto de Investigaciones Jurídicas, UNAM, México / PNPD CAPES no PPGDireito IMED. Linha de pesquisa Mecanismos de efetivação da democracia e da sustentabilidade. Grupo de pesquisa Direito, Novas Tecnologias e Desenvolvimento. E-mail: <magipe@hotmail.com>.

** Pós-Doutora em Direito pela UFSC. Professora do PPGDireito da IMED-Faculdade Meridional. Linha de pesquisa Mecanismos de efetivação da democracia e da sustentabilidade. Grupo de pesquisa Direito, Novas Tecnologias e Desenvolvimento. Grupo de Pesquisa GEDIPI. Professora do IESA e da UFFS. E-mail: < salete.oro.boff@gmail.com>. 


\section{INTRODUCCIÓN}

Dentro de los conceptos de la "producción" y "abastecimiento" de medicamentos, la tendencia actual en la doctrina esboza una idea de innovación inclusiva que denomino "Dinámica Inclusiva del Desarrollo Tecnológico", es decir, un desarrollo de dinámicas con miras a incluir todos los sectores que participan en la cadena productiva de la invención dentro de un política integrada y articulada, caracterizada por las premisas de eficiencia del gasto público y privado en la producción de medicamentos dentro de un conjunto amplísimo de estrategias, así como la garantía de acceso a la salud en igualdad de condiciones y de manera oportuna y la no menos importante distribución de fármacos dentro de una zona geográfica trazada como parte de la mencionada política.

El beneficio último de la 'dinámica inclusiva del desarrollo tecnológico', según la experiencia internacional enfatizada en este apartado en la Argentina, país de altos índices en la PPM, es que en definitiva se logre que exista un instrumento de política pública capaz de remediar y de ser posible homogeneizar, en términos de funcionamiento adecuado, el acceso a la salud en igualdad de condiciones hacia todos los sectores de la población.

Para poder entender mejor lo que significa "suavizar" los derechos de propiedad industrial con miras a la satisfacción de políticas públicas, comenzaremos por abarcar el concepto de licencias obligatorias, también llamadas compulsivas, figura ya más conocida dentro de la citada rama del derecho, que ha cobrado relevancia en importantes acontecimientos del derecho comparado (Brasil, entre los países a citarse).

\section{LICENCIAS OBLIGATORIAS}

Por licencias obligatorias se entiende el permiso que se atribuye a un gobierno para producir un producto o procedimiento patentado o para importarlo, sin el consentimiento del titular de la patente. Se trata de una de las flexibilidades en la protección de las patentes que contempla el Acuerdo de la Organización Mundial del Comercio (OMC) sobre Propiedad Intelectual, a saber, el Acuerdo sobre los Aspectos de los Derechos de Propiedad Intelectual relacionados con el Comercio (ADPIC), a través de la Declaración de Doha de 2001 y sus modificaciones posteriores, en las que se suaviza el derecho de protección de que gozan los fármacos, al reconocer a los países su derecho a tomar medidas tendientes a la protección de la salud pública y la promoción del acceso a las medicinas, conforme lo han reclamado los países en desarrollo y las organizaciones no gubernamentales.

En estricto sentido, a través del contrato de licencia el licenciante autoriza a un tercero - el licenciatario -, con o sin contraprestación, a explotar una invención protegida, mediante el otorgamiento de un derecho de naturaleza personal. ${ }^{1}$ Normalmente vendrá a ser la autoridad gubernamental quien concederá la licencia a empresas o personas distintas del titular de la patente 
a fin de que éstas hagan uso de los derechos de la patente para fabricar, usar, vender o importar el producto o procedimiento protegido sin hacerse necesaria la autorización del titular.

A pesar de hallarse contemplada esta figura en la normativa internacional y de haber sido consecuentemente adoptada por la mayoría de los países adherentes a la $\mathrm{OMC}$ en su legislación interna, su puesta en marcha ha generado controversias sobre todo en el ámbito de la salud ante el caso de emergencias de salud pública.

A priori, la misma doctrina no se muestra unánime en cuanto a su naturaleza jurídica de si se trata de una mera tolerancia o de una contraprestación que asegure un goce económico para el licenciante. A posteriori, se suscitan numerosas dudas a la hora de intentar hacerla efectiva, generándose incertidumbres tanto para el licenciante como para el licenciatario, temores que incluyen hasta problemas de terminología. Por otra parte, una vez celebrado el contrato, los casos de éxito resultan escasos, por lo que la jurisprudencia aún es insuficiente para brindar soluciones sólidas y a largo plazo y, en consecuencia, se regresa errónea e irremediablemente a los principios generales del derecho contractual, en la intención de llegar a solucionar en forma íntegra el problema concreto que se plantea.

De hecho, la doctrina especializada indica el caso de los contratos de licencia y de transferencia de tecnología como un caso típico de incertidumbre en el derecho privado ante la falta de normativa directamente aplicable al tema ${ }^{2}$ que además resulte adecuada y correcta en el sentido de ajustarse a la realidad del país de que se trata, ${ }^{3}$ más allá de a los requerimientos del marco legal internacional, que se limita a estipular conceptos básicos de manera similar y homogénea para todos los países miembros de la OMC.

\section{EL CASO DE LAS LICENCIAS OBLIGATORIAS EN EL CAMPO DE LA SALUD. LA EXPERIENCIA INTERNACIONAL Y LA PROPIA MEXICANA}

En septiembre de 2009 se ha formulado en México una propuesta de reforma del segundo párrafo del artículo 77 de la Ley de Propiedad Industrial, en el sentido de acortar el plazo estipulado para la resolución de la concesión de licencias por la autoridad. La iniciativa no prosperó. ${ }^{4}$

La intención de la propuesta era darle mayor aplicabilidad a la figura de la licencia obligatoria por causa de emergencia nacional y específicamente de enfermedades graves, reduciendo el plazo previsto por el actual segundo párrafo del aludido artículo. ${ }^{5}$

La exposición de motivos daba especial relieve a la emergencia sanitaria con motivo del brote del virus de la influenza, advirtiendo además la presencia de otras enfermedades como el dengue, que ameritan la posibilidad de importar 
medicamentos patentados bajo el régimen de licencias obligatorias, al no contar con capacidad de fabricación suficiente a nivel local, una problemática repetida en países en vías de desarrollo.

Cabe acotar que la esencia de la licencia obligatoria supone hacer siempre subsistir el requisito que establece el primer párrafo del referido artículo 77 de la Ley de Propiedad Industrial mexicana de que las licencias se otorgarán mientras dure la causa de emergencia o de seguridad nacional que haya motivado su celebración ${ }^{6}$ pero olvida el caso de los medicamentos huérfanos, que trataremos más adelante en el capítulo de Unidades Productoras de Medicamentos (UPM) en la Producción Pública de Medicamentos (PPM).

Dentro de la exigua experiencia internacional existente en el campo de las referidas licencias obligatorias, en el ámbito del derecho comparado revisaremos la especial relevancia de la experiencia brasileña con el retroviral denominado efavirenz, producido por la farmacéutica Merck Sharp \& Dohme, considerado en la actualidad como el más eficaz para combatir la infección del SIDA o VIH. A pesar de las fuertes críticas provenientes de la Federación Internacional de la Industria del Medicamento, el gobierno de Brasil, a través de su Presidente en ejercicio Luiz Inácio Lula da Silva, en base a lo que dispone su Ley de Propiedad Industrial 9279/96 en su controvertido artículo 68 que regula las "licencias compulsivas," determinó imponer tal flexibilidad sobre la patente del citado fármaco por considerarla "legítima y necesaria, de interés nacional y demasiado caro", permitiendo con un amplio respaldo del Congreso la importación del genérico producido en laboratorios de la India que se hallan certificados por la Organización Mundial de la Salud (OMS) ${ }^{8}$ quienes ya cuentan con producción del genérico del efavirenz y lo comercializan a un costo inferior que oscila en la tercera parte del producido por el laboratorio titular de la patente. ${ }^{9}$

La medida del Presidente Lula buscaba asegurar el tratamiento de unos 75 mil infectados en el territorio brasileño, siendo este país uno de los mayores compradores mundiales del medicamento, con más de 500 mil infectados de SIDA o VIH. A juicio del gobierno, más allá de demostrar el fracaso en la negociación del precio del fármaco de patente, la medida se convirtió más bien en una prueba de la firmeza gubernamental de apostar por el mercado de los genéricos ante una situación de emergencia nacional.

Cabe destacar que el argumento también se ha apoyado en el tratamiento que actualmente reciben unas 200 mil personas que reciben del Estado un conjunto de 17 medicamentos, de los cuales 8 son fabricados en Brasil dentro del marco de un programa gubernamental de lucha contra la enfermedad que ha recibido un sinnúmero de elogios a nivel internacional.

Como precedente, ya en la Asamblea Mundial de 1999, Brasil había ejercido fuerte presión a fin de que la Organización Mundial de la Salud (OMS) llevara mejor a cabo su labor de control de precios de los medicamentos en todo el mundo y de evaluación en el impacto de las normas sobre patentes de la OMC. 
En abril de 2001, en la reunión anual de la Comisión de Derechos Humanos de las Naciones Unidas fue aprobada por 52 votos a favor, 0 en contra y 1 en abstención (Estados Unidos) la propuesta brasileña que vincula el adecuado acceso a los medicamentos con los derechos humanos fundamentales. Estados Unidos consideró al respecto que tal propuesta era violatoria de las normas internacionales de protección de los derechos de propiedad intelectual. Brasil ya había anunciado en el año 2005 la quiebra de la patente del principio activo kaletra, propiedad del laboratorio Abbot, pero ambas partes pudieron llegar a un acuerdo para la reducción del precio del medicamento.

En el caso del efavirenz, el contrato de licencia se ha llevado a cabo cumpliendo cabalmente el procedimiento previsto a tal efecto en la Declaración de Doha, el cual consta de tres etapas, a saber, la negociación, la declaración de la situación que justifica su utilización y el pago de royalties al titular afectado, que en este caso específico se pactó en la entrega del 1.5\% sobre el valor de importación de los medicamentos similares al efavirenz. ${ }^{10}$

Por otra parte, Tailandia también anunció la imposición de una licencia obligatoria a fin de que los enfermos de SIDA o VIH en este país pudieran recibir tratamiento con el genérico del efavirenz que se produce en laboratorios de la India, país conocido en los últimos tiempos por su alta producción de medicamentos genéricos. ${ }^{11}$ La postura tailandesa se mantuvo a pesar de fuertes presiones provenientes la industria farmacéutica, sentando precedentes en la materia para el caso de emergencias sanitarias. Posteriormente, este país rompió además la patente de otros dos fármacos útiles también en el tratamiento de SIDA o VIH. Otros países como Canadá e Italia cuentan con experiencia en licencias obligatorias sobre productos farmacéuticos y en la década anterior llamó la atención general la decisión del Presidente de Ecuador, Rafael Correa, de emitir en estos días un decreto que derogue por completo las patentes farmacéuticas y agroquímicas de las trasnacionales que tengan efecto en este país, con excepción de las patentes cosméticas, a fin de que todas las medicinas sean producidas en el país y así se logre su abaratamiento, al considerar que la salud es un tema prioritario y primero está el derecho humano antes que "el bolsillo de las trasnacionales," enfatizando el caso de los fármacos que combaten el SIDA o VIH y el cáncer.

Hecho el balance de la actual situación de las licencias obligatorias en el ámbito doméstico y comparado, surge que tal figura presenta varios aspectos positivos, entre los que se cuentan, además del acceso a los medicamentos a un menor precio, el fomento de la competencia y el desarrollo de la producción nacional. Ahora bien, hay que tener muy presente que un país que no reconoce las patentes de las farmacéuticas trasnacionales no contará con la presencia de estos laboratorios en su territorio y, consecuentemente, se verá desabastecido de medicinas esenciales de alta complejidad producidas exclusivamente por ellos en virtud a su tecnología y amplia experiencia en investigación y desarrollo que ostentan y con la que no cuentan los emergentes laboratorios de producción 
local. Por otro lado, con tal medida y al no reconocerse las patentes, tampoco podrá realizarse la importación por la industria local de farmoquímicos necesarios para la fabricación de las medicinas. Todo ello sin mencionar las sanciones de las que podría ser objeto el país por la OMC y la imposibilidad de poder abastecer de medicinas a todo un país con la sola producción nacional, que en el caso de Ecuador alcanza en la actualidad apenas un 22\% del total de ventas. De hecho, se advierte que un crecimiento desmesurado de medicamentos genéricos requeriría en un futuro de un control exhaustivo por parte de la Secretaría de Salud ${ }^{12}$ local.

El caso de Ecuador se presentaría con tal decreto como uno excepcional y hasta violatorio de las normas previstas para la celebración de la licencia obligatoria pues al parecer no contempla la requerida y previa primera etapa de negociación. Además, resulta absurda la idea de generalizar la medida para todas las patentes de medicinas producidas por laboratorios que no sean locales. Al menos, esta no es la función de la licencia obligatoria, la cual se concede para cada caso específico y para atender determinados problemas de salud, por un tiempo determinado, previa justificación por el gobierno de la situación que la motive. La postura que asume este país, a través de su gobernante, deberá encuadrarse en otra figura -inexistente por cierto- que justifique una total supresión de la protección conferida a todo inventor no local de un medicamento. Es el caso extremo de la defensa de la salud pública, la cual va en detrimento del fomento de la investigación y desarrollo, únicas herramientas capaces de propiciar avances tecnológicos y mejores medicinas.

\section{LA RELEVANCIA DE LA NOCIÓN DE SALUD DENTRO DE LAS POLÍTICAS PÚBLICAS: LA PRODUCCIÓN PÚBLICA DE MEDI- CAMENTOS (PPM) Y REDEFINICIÓN DEL CONCEPTO DE BU- ROCRACIA EN EL SISTEMA DE SALUD}

A principios de los años 2000 la moderna doctrina francesa advertía que el ejercicio de la medicina había mutado en su naturaleza con el desarrollo de nuevas especialidades médicas y la multiplicación del número de especialistas, es decir, los médicos. En paralelo, en razón de tal crecimiento y de una oferta de cuidados cada vez más sofisticados según el avance de la ciencia y la tecnología, el volumen de consumo y de gasto médico también ha tenido un aumento significativo. De ello se colige que la extensión de la protección social a toda la población ha sido el sostén de esta evolución: la palabra "salud" pasó a ser actualmente uno de los principales sectores de actividad de los países. ${ }^{13}$ En otras palabras, dentro de las políticas de salud, los resultados revelan que en las últimas décadas tales políticas se vieron orientadas hacia la inversión creciente en tecnologías médicas cada vez más complejas siendo el puntapié inicial y determinante en esta evolución el mismo peso que revisten los profesionales médicos y el progreso de la ciencia médica, como se apuntó.

Dentro de este contexto, la noción de "medicalización de la sociedad", como llama la sociología francesa, ha estado íntimamente relacionada con el 
desarrollo de leyes sobre la protección social. Ya en el siglo XX esta noción traducía en Francia el hecho de que el "modelo médico" se impone en la definición y toma de responsabilidad de numerosos problemas públicos contemporáneos. ${ }^{14}$ De esta manera, en una sociedad medicalizada, el sector salud cobra relevancia como factor de políticas públicas estatales y, dentro de las grandes interrogantes sobre los diversos mecanismos y programas a desarrollar o acentuar dentro de las políticas de salud establecidas, existe la nueva tendencia de la Producción Pública de Medicamentos (PPM en adelante).

Ahora bien, antes de ahondar en el tema propio del artículo que aquí nos ocupa, habrá que establecer el lugar que ocupa el concepto "burocracia" en la administración pública de la salud, tan sólo a modo referencial.

Propio de sistemas normativos, algunos países como Brasil refieren la burocracia como característica destacada dentro del proceso administrativo, aunque en sentido peyorativo y propio del poder público, desde tiempos muy antiguos y características negativas son suyas: paternalismo, nepotismo, corrupción. Sin embargo y paradójicamente, la evolución histórica del proceso administrativo enfatiza la importancia de implementación de procesos burocráticos adecuados a fin de garantizar la manutención de padrones de calidad de los servicios en las estructuras de las instituciones privadas y públicas. La burocracia es, en sentido amplio, un medio de perfeccionamiento de políticas administrativas capaz de alcanzar niveles de eficiencia compatibles con la modernidad. ${ }^{15}$

Ejemplificando dentro del contexto de salud la función de la comentada palabra burocracia, tenemos que la política de salud como directriz que emana del poder público debe abarcar, entre otras funciones principales, la esencial de promover la estructura y el funcionamiento del sistema de los servicios de salud, según lo establece el propio Centro Panamericano de Planificación de la Salud, perteneciente a la Organización Panamericana de la Salud (OPS), siendo sus miembros fundadores Argentina, Brasil, Colombia, Costa Rica, Chile, Ecuador, El Salvador, Honduras, Nicaragua, Panamá, Paraguay, Perú, Uruguay y Venezuela. ${ }^{16}$ Ya en 1972 el documento denominado Tema 27, que abarca el proyecto del Programa del mismo Centro, establecía como objetivo: "Fortalecer la asesoría de la OPS a los gobiernos para perfeccionar sus procesos de planificación de la salud". Luego nombra entre sus actividades: "Programa de investigaciones con los países:... análisis de las relaciones entre salud y el resto del sistema socioeconómico" 17 como una suerte de domesticación del programa, con adecuación a la realidad de cada país miembro y a su normativa propia.

Más adelante, ya en referencia al propio Centro, se establece entre sus responsabilidades una propia de "Investigación", normando sobre la necesidad de apoyar en los países el desarrollo de la misma con miras a elaborar o perfeccionar técnicas de planificación para completar y mejorar la metodología de planificación de la salud, a través del estudio de áreas o variables que deban considerarse y de los países que pudieran elaborarlas, dando además asesoría periódica hasta la fase final de dicha investigación. 
Seguidamente, aparece el apartado de "Información" donde se dispone que toda información que se refiera a la planificación de la salud y a la evolución de los procesos respectivos en los países americanos como de otras regiones debe ser recabada, promovida en su recolección y analizada. Destaco además que la misma debe ser difundida y divulgada a modo de promover, orientar y motivar procesos de planificación y niveles de decisión políticos y administrativos, informando y estimulando a aquellos que realizan la labor directa de planificación en salud, enfatizando las experiencias de terreno conocidas. ${ }^{18}$

Dentro del Programa del Centro puede vislumbrarse la posibilidad de contemplar como política pública de salud nacional la Dinámica Inclusiva de Desarrollo Tecnológico, que se verá explicado más adelante en detalle y sobre lo cual anticipo en este apartado que se describe como una real necesidad de política pública de salud íntegra y que merece ser adecuadamente formulada y plasmada en su planeación, su proyecto y sus resultantes programas que deberán ser coordinados con miras a su ejecución. Esta Dinámica se plasma en esta propuesta a través de Unidades Productoras de Medicamentos (UPM) a nivel público, es decir, la Producción Pública de Medicamentos (PPM).

Volviendo al término burocracia, utilizado en sentido amplio, vendrá entonces en consecuencia a utilizarse para englobar y describir todo aquello que implica el trazado integral del sistema de salud de un país, con sus normativas y organigramas, su contexto social y hasta político, en sentido amplio. En sentido estricto, estamos frente a una burocracia de un sistema de salud circunscripto a una implementación efectiva de las mencionadas UPM.

\section{LA INNOVACIÓN TERAPÉUTICA, LA INVESTIGACIÓN MÉDI- CA CON LA CONTRIBUCIÓN DEL PACIENTE Y LAS UNIDADES PRODUCTORAS DE MEDICAMENTOS (UPM)}

En este campo, la socióloga Renée Fox acentúa la posibilidad de un rol activo del paciente contribuyente al desarrollo de la ciencia médica en el mismo hospital, siendo un caso de referencia la utilización de medicamentos de prueba en hospitales como la cortisona o la ciclosporina, principio activo empleado comúnmente en el trasplante de órganos. Su teoría esboza que el médico está desarrollando investigación en el mismo hospital, a través de su práctica con el paciente y dentro de lo que ella denomina "dilema entre experimentación y terapéutica" pues se trata de probar la eficacia de su innovación a través del mismo paciente con un doble objetivo: curar la enfermedad o tratarla por lo menos y también realizar su propia labor de investigación con el enfermo, quien pasa de ser un sujeto pasivo a ser uno activo desde el momento en que conoce que el procedimiento no sólo persigue su curación o alivio sino además el mismo progreso de la ciencia. ${ }^{19}$

En el caso que ocupa a los medicamentos en los hospitales y su relación con la innovación tecnológica a través de su título de protección, a saber, las 
patentes, la propuesta de la función de una UPM es de naturaleza social en el sentido de que, tal como apunta Oro Boff, un sistema de patentes también puede constituirse en un instrumento de fomento y de erradicación de la pobreza porque estamos frente a un papel considerando dentro de un conjunto más amplio de medidas políticas nacionales de desarrollo tecnológico y obedeciendo a los mismos intereses del país. En efecto, tal como ella lo sostiene, diseminar la cultura de salvaguarda de los derechos de propiedad intelectual, priorizando sobre todo la disponibilidad de recursos y garantizando una infraestructura necesaria, parece ser el camino para que pensar que la propiedad intelectual pueda contribuir al desarrollo económico y social. ${ }^{20}$

La propiedad intelectual, importante mecanismo para la protección de invenciones derivadas del intelecto humano es un área diferenciada de la propiedad material y hoy en día cuenta con un importante estímulo cuando se relaciona con la utilidad colectiva, aquí incluidas las invenciones. ${ }^{21}$

Como factor de puesta en marcha del punto anterior en específico así como en general de lo hasta aquí expuesto, es preciso poner en conocimiento una teoría desarrollada en Argentina, país que más se ha preocupado en el tema de la PPM. Esta teoría ha sido recientemente en ese país por especialistas en estudios sociales de tecnología e innovación ${ }^{22}$ que propone abordar la PPM con un enfoque de tecnología organizacional y política pública y como un instrumento que busca como fin dinamizar procesos de desarrollo inclusivo.

Hablar de desarrollo inclusivo significa despuntar la ciencia y la tecnología propia de una región y subsecuentemente de un país pero en esferas que van más allá de la privada o la mixta. Nos referimos a la PPM a través del trabajo articulado de laboratorios públicos y de farmacias hospitalarias y es que, ya en los albores del año 2000, el hospital público ha pasado de ser durante siglos el asilo de los pobres a convertirse en una institución prototipo de las sociedades modernas, lugar de referencia privilegiada en la investigación médica y en el desarrollo de técnicas de punta. ${ }^{23}$

En Argentina, la cuestión va más allá de la ya compleja provisión de medicamentos, enfocándose a puntos estratégicos que plantea actualmente el sector salud. ${ }^{24}$

- La política pública integrada, como factor en sí mismo y como factor capaz de desarrollar políticas públicas transformadoras dentro de la 'dinámica inclusiva del desarrollo tecnológico'.

- La eficiencia del gasto público y privado que se genera en la producción de medicamentos, lo cual implica un conjunto amplísimo de estrategias a desarrollar.

- Una garantía de acceso a la salud en igualdad de condiciones para todos.

- El factor de la innovación con su desafío de generar dinámicas con miras al desarrollo inclusivo. ${ }^{25}$

A partir de esos cuatro puntos estratégicos mencionados que plantea el sector salud citan los especialistas tres preguntas-problema: ${ }^{26}$ 
- ¿Qué capacidad tiene el actual sector público productor de medicamentos y cuál es la relación entre política de salud y mercado de medicamentos?

- Como factor de implementación y de transformación, ies posible configurar una política integral de producción pública de medicamentos que conviva con la compra privada?

- ¿Bajo qué condiciones una política pública de PPM puede desplegar dinámicas concretas de desarrollo inclusivo?

El panorama debe ser visto el sector general tanto con sus complejidades como con sus oportunidades.

Existe una fuerte crítica al sistema privado y a la industria farmacéutica que éste desarrolla por adueñarse del presupuesto general destinado al sector salud y de la cadena de provisión de medicamentos, pero esto no es visto desde la raíz del problema: si se tuviera un mayor escenario presupuestario destinado a la eficiencia innovadora por las UPM y una participación concreta y eficiente de éstas dentro del aparato legal y regulatorio, podría verse más abierta gran parte de tal hegemonía privada.

La pregunta resaltante que hemos de hacernos es icuál es la importancia que adquiere el mercado de los medicamentos y su dinámica dentro del concepto de gasto en salud? Existen muchas y conocidas inconsistencias como por ejemplo la baja en la capacidad de compra frente a la permanencia alcista de la capacidad de venta lo cual es producto de la propia estructura y diseño del sistema de salud y su consecuente dinámica. ${ }^{27}$

El secreto para el desarrollo inclusivo las UPM pareciera radicar en los conceptos de Presupuesto + Participación Eficiente de la UPM, no respecto la eterna discusión entre el sector genérico y el innovador sino frente a la apropiación y hegemonía de todo el sector farmacéutico privado en el presupuesto público, dándose en consecuencia una altísima concentración en laboratorios privados nacionales y trasnacionales con el consecuente control de precios determinado por el sector dominante resultando la fórmula: a mayor concentración, mayor control de precios.

Las UPM conforman el sector de la PPM y se subdividen en laboratorios públicos y en farmacias hospitalarias. Ambos tipos producen medicamentos pero dirigidos a un público distinto ya que las segundas destinan sus productos generalmente a los pacientes a cuyo hospital pertenecen, mientras que los laboratorios públicos surten medicamentos a una extensa red de sistemas de salud a lo largo del territorio del país, abarcando hospitales públicos o privados, planes y programas de provisión pública de medicamentos o inclusive venta minorista en farmacias. ${ }^{28}$

Es de tener en cuenta además que entre esos laboratorios podemos encontrar lo que dependen del gobierno nacional, de universidades nacionales, de gobiernos provinciales e incluso de municipios. ${ }^{29}$ 
Analizando lo que contempla el estudio de las farmacias hospitalarias, en la PPM, basándose en la Resolución Ministerial argentina 286/08, la salud es un derecho y el medicamento un bien social siendo una función del Estado garantizar la accesibilidad y ordenar la distribución de recursos y el aprovechamiento de capacidades instaladas y de recursos humanos. ${ }^{30}$

Frente al problema del acceso a los medicamentos como producto por ejemplo de una crisis, siempre es el esfuerzo público, a través del Estado, el que acaba respondiendo con medidas alternativas, por ejemplo, la provisión gratuita de medicamentos en determinado tiempo y lugar a través de planes concretos ${ }^{31}$. Una vez recuperada la actividad económica, la producción privada vuelve a estar en alza, incrementando su facturación (control de precios en consecuencia) y trayendo como consecuencia que la crisis haya sido finalmente absorbida en forma total por el sector público. ${ }^{32}$

Las propuestas son varias y pertenecerían a otro capítulo pero entre las posibles soluciones cobra especial relevancia UPM. Garantizar el abastecimiento oportuno de medicamentos, si bien forma parte de la agenda pública y es una obligación del Estado, esto no implica forzosamente que estos bienes deban ser comprados al sector privado. En consecuencia, debería vincularse la política pública a una estrategia de producción y no de compra directa, conforme explican los estudiosos argentinos. ${ }^{33}$

La elaboración de especialidades medicinales por laboratorios públicos acabaría con el pensamiento radical de la naturaleza del medicamento como un bien de mercado, transformándolo en un bien social ${ }^{34}$ pero existen realidades de las UPM, que se enumeran someramente a continuación.

1) Producción y abastecimiento: En la Argentina, a pesar de contar con un diagrama trazado en la cobertura geográfica de la UPM a través de laboratorios que cuentan con habilitación de sendos permisos de instalaciones y de producción por parte del organismo regulador competente, a saber, la Administración Nacional de Medicamentos, Alimentos y Tecnología Médica (ANMAT), cuya finalidad última es la circulación de los productos en el territorio nacional para su venta minorista, en la práctica resulta que varios de ellos no alcanzan la cobertura nacional efectiva (problemas de distribución) y entre las limitantes de la problemática en la distribución efectiva del medicamento, la misma regulación encabeza la lista que tiene que ver con el acondicionamiento de las instalaciones de las UPM al requerimiento normativo.

2) Patentes: sin entrar en detalle al respecto, me centro en específico en la siguiente limitante que se arguye acerca de la distribución efectiva de los medicamentos elaborados por las UPM y que tiene que ver con la fuerte crítica que recibe la protección del medicamento a través de la figura de la propiedad industrial que le corresponde: la patente. En efecto, las UPM fabrican los medicamentos con principios activos cuyas patentes se encuentran vencidas y cuentan con restricción para elaborar medicamentos con patente vigente. 
El argumento de la protección de la patente se encuentra desarrollado ampliamente en la doctrina actual pero encuentra un gran vacío en el caso de la PPM, caso en que la producción - y distribución aludida - del medicamento por las UPM debiera considerar políticas de excepción, máxime si se trata de medicamentos huérfanos, a través de oportunas previsiones legales como las licencias obligatorias, sobre lo cual las legislaciones aún encuentran importantes vacíos a la hora de ponerlas en marcha.

3) Tecnología e infraestructura local: sigue siendo un obstáculo importante el hecho de que los principios activos o farmoquímicos sean adquiridos en el exterior por falta de producción local suficiente que se traduce en una capacidad inventiva limitada por parte de la industria nacional importadora de aquellas fórmulas, dicho

$\mathrm{Al}$ respecto esgrimen los autores como causa del problema las racionalidades que imperan en las políticas y programas de ciencia y tecnología en el sentido de que éstas responden al modelo lineal de innovación. Así, si bien existe un número importante de institutos y centros de investigación médica, todavía cuentan con bajos niveles de interacción con las unidades de producción. Aquí cobra una excepción importante los laboratorios que pertenecen a las universidades nacionales sin que por ello el producido global de la dinámica innovativa del país resulte más feliz por tal excepción. ${ }^{39}$

4) UPM existentes: Parte de esta política lineal debe también culpa a la concentración excesiva en manos de algunas UPM estatales. En Argentina, se citan veinte UPM como parte integrante de la Red Nacional de Productores Públicos de Medicamentos, siendo su objetivo la producción conjunta de medicamentos de manera coordinada pero cuyos bajos resultados saltan a la vista por falta de acciones concretas en materia de producción y también de posicionamiento de la producción pública entre los temas principales de la agenda política del sector salud pública.

No se puede dejar de mencionar sin embargo que en el año 2011, en un esfuerzo más defensivo que proactivo de las UPM y del Grupo de Gestión de Políticas de Estado en Ciencia y Tecnología, se obtuvo la Ley 26.688 que declaró de interés nacional la investigación y producción pública de medicamentos, sus materias primas, las vacunas y los productos médicos. ${ }^{40}$ Esta ley argentina aún carece de reglamento pero puede resultar un sugerente a la normativa de otros países en la materia por sus líneas estratégicas de acción, entre las que se mencionan:

- La definición de prioridades en líneas estratégicas de producción según perfiles epidemiológicos y estacionales de cada región.

- La promoción de investigación, desarrollo y producción de medicamentos huérfanos. ${ }^{41}$

Entre los principales principios activos elaborados por laboratorios públicos se encuentran analgésicos y antibióticos de uso habitual y también 
drogas utilizadas para tratar enfermedades crónicas. La provincia con mayor abastecimiento de medicamentos de producción pública es la de Santa Fe y le siguen San Luis y Río Negro. ${ }^{42}$ En el año 2014 se planificó un financiamiento por la Agencia Nacional de Promoción Científica y Tecnológica del Fondo Argentino Sectorial (FONARSEC) para la producción pública de medicamentos tuberculostáticos integrando el trabajo conjunto de la Universidad del Litoral y el Laboratorio Industrial Farmacéutico S.E. (LIF) cubriendo áreas estratégicas y de vacancia en la provisión de especialidades medicinales prioritarias. ${ }^{43}$

\section{PRINCIPALES PRINCIPIOS ACTIVOS ELABORADOS POR LABO- RATORIOS PÚBLICOS EN ARGENTINA ${ }^{44}$}

Según se enlista seguidamente estos son los principales principios activos producidos actualmente por laboratorios públicos

\begin{tabular}{|l|l|c|}
\hline \multicolumn{1}{|c|}{ Principio activo } & \multicolumn{1}{c|}{ Acción terapéutica } & Cantidad de laboratorios \\
\hline Paracetamol & Analgésico & 12 \\
\hline Enalapril & Antihipertensivo & 11 \\
\hline Furosemida & Diurético & 11 \\
\hline Glibenclamina & Hipoglucemiante & 11 \\
\hline Diazepam & Ansiolítico & 10 \\
\hline Ibuprofeno & Antiinflamatorio & 10 \\
\hline Mebendazol & Antiparasitario & 9 \\
\hline Permetrina & Pediculicida & 9 \\
\hline Amoxicilina & Antibiótico & 8 \\
\hline Atenolol & Antihipertensivo & 8 \\
\hline Diclofenaco & Antiinflamatorio & 8 \\
\hline Ranitidina & Antiulceroso & 8 \\
\hline Fenobarbital & Anticonvulsivo & 7 \\
\hline Metformina & Hipoglucemiante & 7 \\
\hline Metodopramina & Antiemético & 7 \\
\hline
\end{tabular}

\section{CONCLUSIONES}

Dentro de la "Dinámica Inclusiva del Desarrollo Tecnológico", la Producción Pública de Medicamentos (PPM) llevada a cabo a través de sus Unidades Productoras juega un rol preponderante en la agenda pública del sector salud del país. Cuando es puesta en marcha de manera coordinada y positiva, conlleva en sí misma fines de inclusión y desarrollo social y productivo y es un instrumento de política pública de salud integrada y dinámica innovativa con capacidad de producir bajo demanda específica obedeciendo a un trazado territorial nacional 
prestablecido, con límites demográficos. De esta manera, cumple la función de asegurar la provisión y distribución adecuadas a la población y desarrollar los medicamentos huérfanos.

Visto como lo que es y aunque le pese la realidad de la desventura de los problemas propios del necesario aparato burocrático legal, la PPM es una opción a la elaboración tradicional privada de medicamentos, llevada a cabo por laboratorios nacionales, con principios activos que sean accesibles en el mercado nacional y extranjero. Es una herramienta que conlleva en sí misma la noble acción a la que está destinado un fármaco, que es no es otra que cumplir con un bien social de acceso a la salud más allá de su valor patrimonial, sin desmerecer la propiedad intelectual de la patente de la que goza el medicamento, lo cual puede lograrse con un Plan de Acción que prevea no sólo mecanismos legislativos oportunos sino por sobre todo, estrategias de puesta en marcha en los distintos ciclos de la innovación farmacéutica vista, en sus distintas fases y hasta llegar a manos del consumidor final.

Finalmente, en cuanto al primer tema que hemos abordado, dejamos por sentada la tarea positiva que conllevaría una licencia obligatoria bien estructurada, celebrada y fundamentalmente concedida respetando cualesquiera de sus dos principales finalidades, a saber, la de salvar una situación de emergencia nacional o la de garantizar su efectiva explotación si ésta no se ha producido en el territorio. Sin embargo, no se debe olvidar que muchas veces la promoción de esta figura encubre una finalidad encubierta, en el sentido de que más allá de garantizar la salud pública lo que realmente busca es facilitar y patrocinar la copia ilegal de medicamentos, lo cual constituye un flagrante ataque a la propiedad intelectual que se traduce en falta de seguridad jurídica para el inversor, provocando este hecho un gran desaliento a la inversión extranjera en cualquier país del mundo.

\section{REFERÊNCIAS}

ADAM, Philippe; HERZLICH, Claudine. Sociologie de la maladie et de la médecine. Saint-Jean de Braye: Editorial Armand Colin, 2010.

ARGENTINA. Ministerio de Ciencia, Tecnología e Innovación Productiva. Nuevo financiamiento para producción pública de medicamentos tuberculostáticos. 2014. Disponible en internet en: < http://www.mincyt.gob.ar/ noticias/nuevo-financiamiento-para-produccion-publica-de-medicamentos-tuberculostaticos-10181>. Consultado el: 7 sept. 2016.

ARGENTINA. Ministerio de Economía y Finanzas Públicas. Secretaría de Política Económica y Planificación del Desarrollo. Subsecretaría de Planificación Económica. Dirección Nacional de Planificación Regional. Dirección Nacional de Planificación Sectorial. Complejo Farmacéutico. Serie Complejos Productivos. 2015. Disponible en internet en: <http://www.economia.gob.ar/ peconomica/docs/Complejo_Farmaceutico.pdf >. Consultado el: 7 sept. 2016. 
ARGENTINA. Ley 26.688 que declara de interés nacional la investigación y producción pública de medicamentos, sus materias primas, las vacunas y los productos médicos.

BOFF, Salete Oro. Propriedade intelectual e desenvolvimento. Inovação, gestão e transferência tecnológica. Passo Fundo: IMED Editora, 2009.

BOFF, Salete Oro; LIPPSTEIN, Daniela. Biodiversidade, conhecimentos tradicionais e propriedade intelectual: um caminho para o desenvolvimiento sustentável. E: BOFF, Salete Oro et al. (Coord.). Novas tecnologias, direitos intelectuais e políticas públicas. São Paulo: Letras jurídicas, 2015. v. 2.

CAMPOS, Juarez De Queiroz; PRESOTO, Lúcia Helena. A burocracia na administração de saúde. São Paulo: Editora Jotacê, 2002.

DAHER, Mauricio Jalife. Régimen de licencias obligatorias de patentes en el campo de la salud. En: SESMA, Ingrid Brena (Coord.). Salud y Derecho. Memoria del Congreso Internacional de Culturas y Sistemas Jurídicos Comparados. Ciudad de México: Instituto de Investigaciones Jurídicas de la Universidad Nacional Autónoma de México, 2005.

DE LAS CUEVAS, Guillermo Cabanellas. Contratos de licencia y de transferencia de tecnología en el derecho privado. Buenos Aires: Editorial Heliasta, 1994.

FOX, Renée. Experiment Perilous, Physicians and Patients Facing the Unknown. 1959. The free Press. Edición en internet: < https://books.google. com.br/books? $\mathrm{id}=\mathrm{VjN}-$ al61ZoC\&pg $=\mathrm{PA} 2 \& \mathrm{lpg}=\mathrm{PA} 2 \& \mathrm{dq}=\mathrm{ren} \% \mathrm{C} 3 \% \mathrm{~A} 9 \mathrm{e}$ + fox,+ experiment + perilous, + physicians + and + patients + facing + the + un known, + The + free + Press,$+1959 \&$ source $=$ bl\&ots $=$ N7CmOhODB\&sig $=$ gmBhAbW1NDpDnjuHxHoyB10akbI\&hl=es\&sa $=$ X $\&$ ved $=0$ ahUKEwjOuv nDof7OAhUBDZAKHaCWAN8Q6AEIJjAB\#v $=$ onepage $\& q=$ ren $\% C 3 \% A$ 9e\%20fox $\% 2$ C $\% 20$ experiment $\% 20$ perilous $\% 2$ C $\% 20$ physicians $\% 20$ and $\% 20$ patients\%20facing\%20the\%20unknown\%2C\%20The\%20free\%20Press\%2C\%20 1959\&f=false >. Consultado el: 7 sept. 2016.

HOYA, Arturo. Producción Pública de Medicamentos: una respuesta a los medicamentos huérfanos pediátricos. In: CONGRESO ARGENTINO, 14 y CONGRESO SUDAMERICANO DE FARMACIA HOSPITALARIA, 3., 2014, Salta. Anais... Salta: Asociación Argentina De Farmacéuticos De Hospital, 2014. Disponible en: <http://www.aafhospitalaria.org.ar/imagenes/ descargas/2014-6-b.pdf>. Consultado el: 7 sept. 2016.

INSTITUTO ESPAÑOL DE COMERCIO EXTERIOR (ICEX). Oficina Económica y Comercial de la Embajada de España en Brasilia. Patentes y acceso a los medicamentos. 2009. Disponible en: <http://www.fedeto.es/area_internacional/marco_politico_datos_brasil.pdf>. Consultada en: oct. 2009.

JOLIET, René. Der Patentlizenzvertrag im belgischen und französischen Zivilrecht. Munich: GRUR Int., 1982. 
ORGANIZACIÓN PANAMERICANA DE LA SALUD (OPS). Tema 27 del proyecto del programa. 1972. Disponible en: <http://iris.paho.org/xmlui/ bitstream/handle/123456789/5924/

49176.pdf?sequence $=1$ \&isAllowed $=y>$. Consultada en: 7 sept. 2016.

SANTOS, Guillermo; BECERRA, Lucas. La producción pública de medicamentos como parte de una estrategia de política de salud integrada. Reflexiones y recomendaciones para el desarrollo inclusivo en salud. En: THOMAS, Hernán; SANTOS, Guillermo (Coord.). Tecnologías para incluir. Ocho análisis socio-técnicos orientados al diseño estratégico de artefactos y normativas. Buenos Aires: IESCT y Universidad Nacional de Quilmes y Lenguaje Claro, 2016. Agenda CTD.

1 JOLIET, René. Der Patentlizenzvertrag im belgischen und französischen Zivilrecht. Munich: GRUR Int., 1982. p. 294.

2 DE LAS CUEVAS, Guillermo Cabanellas. Contratos de licencia y de transferencia de tecnología en el derecho privado. Buenos Aires: Editorial Heliasta, 1994. p. 12.

3 El derecho comparado ofrece soluciones limitadas en la materia pues habrá de tenerse en cuenta la específica y particular estructura económica del país en que se celebre el contrato.

4 El artículo ya había sido reformado en el año 2004 y anteriormente en 1994. La iniciativa de reforma del segundo párrafo del artículo 77 hecha por el Senador Adolfo Toledo Infanzón del Partido Revolucionario Institucional (PRI), de fecha 8 de septiembre de 2009, rezaba textualmente : "En los casos de enfermedades graves que sean causa de emergencia o atenten contra la seguridad nacional, el Consejo de Salubridad General hará la declaratoria de atención prioritaria, por iniciativa propia o a solicitud por escrito de instituciones nacionales especializadas en la enfermedad, que sean acreditadas por el Consejo, en la que se justifique la necesidad de atención prioritaria, en esta se ordenará el otorgamiento de licencias de utilidad pública. Publicada la declaratoria del Consejo en el Diario Oficial de la Federación, las empresas farmacéuticas podrán solicitar la concesión de una licencia de utilidad pública al Instituto y éste la otorgará, en un plazo no mayor a tres días, a partir de la fecha de presentación de la solicitud ante el Instituto."

5 La propuesta establecía un plazo máximo de 3 días para que la autoridad se pronuncie, dejando sin efecto la disposición vigente, que es su parte pertinente reza : “... a la brevedad que el caso lo amerite de acuerdo con la opinión del Consejo de Salubridad General en un plazo no mayor a 90 días...” y que además exige que sea audita parte. Cabe destacar que el texto vigente extrañamente tampoco incluye la frase expresamente propuesta en la iniciativa de reforma de que en los casos aludidos el objetivo de la orden será el "otorgamiento de licencias de utilidad pública". Cfr. Artículo 77 de la Ley de Propiedad Industrial vigente.

6 Artículo 77 LPI México, primer párrafo : "Por causas de emergencia o seguridad nacional y mientras duren éstas, incluyendo enfermedades graves declaradas de atención prioritaria por el Consejo de Salubridad General, el Instituto, por declaración que se publicará en el Diario Oficial de la Federación, determinará que la explotación de ciertas patentes se haga mediante la concesión de licencias de utilidad pública, en los casos en que, de no hacerlo así, se impida, entorpezca o encarezca la producción, prestación o distribución de satisfactores básicos o medicamentos para la población."

7 Estados Unidos denunció ante a la $\mathrm{OMC}$ el artículo 68 de la ley de patentes brasileña por considerar que era violatorio del ADPIC, requiriendo ante tal organismo un Panel de Resolución de Conflictos con el objeto de que éste pueda dirimir las diferencias entre estos dos países. Brasil se defendió ante la OMC alegando justificación en la medida por el alto número existente de enfermos de SIDA o VIH y, en consecuencia, considerar un caso de emergencia sanitaria nacional. Finalmente, el 25 de junio de 2001 Estados Unidos retiró ante la OMC la solicitud del panel.

8 Entre esos laboratorios genéricos están Ranbaxy, Cipla y Aurobindo.

9 Mientras Merck cotizaba en 1.65 USD cada unidad del fármaco en territorio brasileño el costo por unidad del genérico fabricado en la India es de 0.44 USD. El tratamiento por paciente tiene un costo por año de 580 USD utilizando el medicamento de patente mientras que el tratamiento utilizando el genérico alcanza la suma de 165 USD anuales, es decir, menos de un tercio, lo cual implica un ahorro 
de 240 millones USD hasta el año 2012, fecha en que expira la patente. Antes de la imposición de la licencia obligatoria sobre el fármaco, Lula había mantenido dos negociaciones con el laboratorio titular, en las que participó además el gobierno norteamericano. Brasil rechazó en la segunda de ellas la oferta de comercializar el producto con un $30 \%$ menos del precio fijado originalmente por parecerle insuficiente y considerar que al menos debía reducirse en un $60 \%$. En las negociaciones, Lula propuso el mismo precio pagado por Tailandia, que es de 0.65 USD por cada comprimido del fármaco en dosis de 600 miligramos, lo cual no fue aceptado por Merck.

10 INSTITUTO ESPAÑOL DE COMERCIO EXTERIOR (ICEX). Oficina Económica y Comercial de la Embajada de España en Brasilia. Patentes y acceso a los medicamentos. 2009. Disponible en: <http:// www.fedeto.es/area_internacional/marco_politico_datos_brasil.pdf >. Consultada en: oct. 2009.

11 La organización no gubernamental Médicos Sin Fronteras utiliza casi en su totalidad medicamentos producidos en la India para tratar a enfermos de SIDA o VIH en 30 países del mundo.

12 DAHER, Mauricio Jalife. Régimen de licencias obligatorias de patentes en el campo de la salud. En: SESMA, Ingrid Brena (Coord.). Salud y Derecho. Memoria del Congreso Internacional de Culturas y Sistemas Jurídicos Comparados. Ciudad de México: Instituto de Investigaciones Jurídicas de la Universidad Nacional Autónoma de México, 2005. p. 332.

13 ADAM, Philippe; HERZLICH, Claudine. Sociologie de la maladie et de la médecine. Saint-Jean de Braye: Editorial Armand Colin, 2010. p. 36.

14 Citando algunos padecimientos a título de ejemplo: alcoholismo, enfermedades mentales, drogas.

15 CAMPOS, Juarez De Queiroz; PRESOTO, Lúcia Helena. A burocracia na administração de saúde. São Paulo: Editora Jotacê, 2002. p. 5.

16 ORGANIZACIÓN PANAMERICANA DE LA SALUD (OPS). Tema 27 del proyecto del programa. 1972. p. 8. Disponible en: <http://iris.paho.org/xmlui/bitstream/handle/123456789/5924/49176. pdf? sequence $=1$ \&isAllowed $=\mathrm{y}>$. Consultada en: 7 sept. 2016.

17 ORGANIZACIÓN PANAMERICANA DE LA SALUD (OPS). Tema 27 del proyecto del programa. 1972. p. 9. Disponible en: <http://iris.paho.org/xmlui/bitstream/handle/123456789/5924/49176. pdf? sequence $=1$ \&isAllowed $=y>$. Consultada en: 7 sept. 2016.

18 ORGANIZACIÓN PANAMERICANA DE LA SALUD (OPS). Tema 27 del proyecto del programa. 1972. p. 13. Disponible en: <http://iris.paho.org/xmlui/bitstream/handle/123456789/5924/49176. pdf? sequence $=1$ \&isAllowed $=y>$. Consultada en: 7 sept. 2016.

19 FOX, Renée. Experiment perilous, physicians and patients facing the unknown. 1959. The free Press. Edición en internet: $<$ https://books.google.com.br/books?id $=\mathrm{VjN}$-_al6lZoC\&pg $=\mathrm{PA} 2 \& l p g=\mathrm{PA} 2 \& \mathrm{dq}$ $=$ ren $\% \mathrm{C} 3 \% \mathrm{~A} 9 \mathrm{e}+$ fox,+ experiment + perilous,+ physicians + and + patients + facing + the + unknown,+ The + free + Press,$+1959 \&$ source $=$ bl\&ots $=$ N7CmOhODB\&sig $=$ gmBhAbW1NDpDnjuHxHoyB10a $\mathrm{kbI \& hl}=\mathrm{es} \& \mathrm{sa}=\mathrm{X} \& \mathrm{ved}=0 \mathrm{ahUKEwjOuvnDof7OAhUBDZAKHaCWAN8Q6AEIjjAB} \#_{\mathrm{v}}=$ onepage $\&$ $\mathrm{q}=$ ren $\% \mathrm{C} 3 \% \mathrm{~A} 9 \mathrm{e} \% 20$ fox\%2C\%20experiment $\% 20$ perilous\%2C\%20physicians\%20and\%20patients $\% 20$ facing\%20the\%20unknown\%2C\%20The\%20free\%20Press\%2C\%201959\&f=false >. Consultado el: 7 sept. 2016.

20 BOFF, Salete Oro. Propiedade intelectual e desenvolmiento. Inovação, gestão e transferência tecnológica. Passo Fundo: IMED Editora, 2009. p. 57.

21 BOFF, Salete Oro; LIPPSTEIN, Daniela. Biodiversidade, conhecimentos tradicionais y propiedade intelectual: um caminho para o desenvolvimiento sustentável. En: BOFF, Salete Oro et al. (Coord.). Novas tecnologias, direitos intelectuais e políticas públicas. São Paulo: Letras jurídicas, 2015. v. 2. p. 30.

22 Santos, Guillermo y Becerra, Lucas, Investigadores del Área de Estudios Sociales de la Tecnología y la Innovación del Instituto de Estudios sobre la Ciencia y la Tecnología (UNQ) sobre cuyo trabajo se basa el presente artículo.

23 ADAM, Philippe; HERZLICH, Claudine. Sociologie de la maladie et de la médecine. Saint-Jean de Braye: Editorial Armand Colin, 2010. p. 37.

24 SANTOS, Guillermo; BECERRA, Lucas. La producción pública de medicamentos como parte de una estrategia de política de salud integrada. Reflexiones y recomendaciones para el desarrollo inclusivo en salud. En: THOMAS, Hernán; SANTOS, Guillermo (Coord.). Tecnologías para incluir. Ocho análisis socio-técnicos orientados al diseño estratégico de artefactos y normativas. Buenos Aires: IESCT y Universidad Nacional de Quilmes y Lenguaje Claro, 2016. Agenda CTD. p. 251. 
25 SANTOS, Guillermo; BECERRA, Lucas. La producción pública de medicamentos como parte de una estrategia de política de salud integrada. Reflexiones y recomendaciones para el desarrollo inclusivo en salud. En: THOMAS, Hernán; SANTOS, Guillermo (Coord.). Tecnologías para incluir. Ocho análisis socio-técnicos orientados al diseño estratégico de artefactos y normativas. Buenos Aires: IESCT y Universidad Nacional de Quilmes y Lenguaje Claro, 2016. Agenda CTD. p. 251.

26 SANTOS, Guillermo; BECERRA, Lucas. La producción pública de medicamentos como parte de una estrategia de política de salud integrada. Reflexiones y recomendaciones para el desarrollo inclusivo en salud. En: THOMAS, Hernán; SANTOS, Guillermo (Coord.). Tecnologías para incluir. Ocho análisis socio-técnicos orientados al diseño estratégico de artefactos y normativas. Buenos Aires: IESCT y Universidad Nacional de Quilmes y Lenguaje Claro, 2016. Agenda CTD. p. 251.

27 SANTOS, Guillermo; BECERRA, Lucas. La producción pública de medicamentos como parte de una estrategia de política de salud integrada. Reflexiones y recomendaciones para el desarrollo inclusivo en salud. En: THOMAS, Hernán; SANTOS, Guillermo (Coord.). Tecnologías para incluir. Ocho análisis socio-técnicos orientados al diseño estratégico de artefactos y normativas. Buenos Aires: IESCT y Universidad Nacional de Quilmes y Lenguaje Claro, 2016. Agenda CTD. p. 255-257.

28 SANTOS, Guillermo; BECERRA, Lucas. La producción pública de medicamentos como parte de una estrategia de política de salud integrada. Reflexiones y recomendaciones para el desarrollo inclusivo en salud. En: THOMAS, Hernán; SANTOS, Guillermo (Coord.). Tecnologías para incluir. Ocho análisis socio-técnicos orientados al diseño estratégico de artefactos y normativas. Buenos Aires: IESCT y Universidad Nacional de Quilmes y Lenguaje Claro, 2016. Agenda CTD. p. 259.

29 SANTOS, Guillermo; BECERRA, Lucas. La producción pública de medicamentos como parte de una estrategia de política de salud integrada. Reflexiones y recomendaciones para el desarrollo inclusivo en salud. En: THOMAS, Hernán; SANTOS, Guillermo (Coord.). Tecnologías para incluir. Ocho análisis socio-técnicos orientados al diseño estratégico de artefactos y normativas. Buenos Aires: IESCT y Universidad Nacional de Quilmes y Lenguaje Claro, 2016. Agenda CTD. p. 259.

30 HOYA, Arturo. Producción Pública de Medicamentos: una respuesta a los medicamentos huérfanos pediátricos. In: CONGRESO ARGENTINO, 14 y CONGRESO SUDAMERICANO DE FARMACIA HOSPITALARIA, 3., 2014, Salta. Anais... Salta: Asociación Argentina De Farmacéuticos De Hospital, 2014. Disponible en: <http://www.aafhospitalaria.org.ar/imagenes/descargas/2014-6-b.pdf>. Consultado el: 7 sept. 2016.

31 Como ejemplo se cita el Plan Remediar del año 2002 a través de la provisión de medicamentos en forma gratuita para 15 millones de personas.

32 SANTOS, Guillermo; BECERRA, Lucas. La producción pública de medicamentos como parte de una estrategia de política de salud integrada. Reflexiones y recomendaciones para el desarrollo inclusivo en salud. En: THOMAS, Hernán; SANTOS, Guillermo (Coord.). Tecnologías para incluir. Ocho análisis socio-técnicos orientados al diseño estratégico de artefactos y normativas. Buenos Aires: IESCT y Universidad Nacional de Quilmes y Lenguaje Claro, 2016. Agenda CTD. p. 257, 258.

33 SANTOS, Guillermo; BECERRA, Lucas. La producción pública de medicamentos como parte de una estrategia de política de salud integrada. Reflexiones y recomendaciones para el desarrollo inclusivo en salud. En: THOMAS, Hernán; SANTOS, Guillermo (Coord.). Tecnologías para incluir. Ocho análisis socio-técnicos orientados al diseño estratégico de artefactos y normativas. Buenos Aires: IESCT y Universidad Nacional de Quilmes y Lenguaje Claro, 2016. Agenda CTD. p. 257, 258.

34 SANTOS, Guillermo; BECERRA, Lucas. La producción pública de medicamentos como parte de una estrategia de política de salud integrada. Reflexiones y recomendaciones para el desarrollo inclusivo en salud. En: THOMAS, Hernán; SANTOS, Guillermo (Coord.). Tecnologías para incluir. Ocho análisis socio-técnicos orientados al diseño estratégico de artefactos y normativas. Buenos Aires: IESCT y Universidad Nacional de Quilmes y Lenguaje Claro, 2016. Agenda CTD. p. 281.

35 Los laboratorios, tanto públicos como privados, para acceder al tránsito federal de su producción deben habilitar sus plantas y certificar sus productos ante ANMAT.

36 Excepto el caso de medicamentos esenciales huérfanos, es decir, no provisto en el mercado interno por los laboratorios privados detentores de la patente sobre lo cual se ha hecho especial énfasis en lo de uso pediátrico.

37 SANTOS, Guillermo; BECERRA, Lucas. La producción pública de medicamentos como parte de una estrategia de política de salud integrada. Reflexiones y recomendaciones para el desarrollo inclusivo en salud. En: THOMAS, Hernán; SANTOS, Guillermo (Coord.). Tecnologías para incluir. Ocho análisis socio-técnicos orientados al diseño estratégico de artefactos y normativas. Buenos Aires: IESCT y Universidad Nacional de Quilmes y Lenguaje Claro, 2016. Agenda CTD. p. 261, 262.

38 SANTOS, Guillermo; BECERRA, Lucas. La producción pública de medicamentos como parte de una 
estrategia de política de salud integrada. Reflexiones y recomendaciones para el desarrollo inclusivo en salud. En: THOMAS, Hernán; SANTOS, Guillermo (Coord.). Tecnologías para incluir. Ocho análisis socio-técnicos orientados al diseño estratégico de artefactos y normativas. Buenos Aires: IESCT y Universidad Nacional de Quilmes y Lenguaje Claro, 2016. Agenda CTD. p. 261, 262.

39 SANTOS, Guillermo; BECERRA, Lucas. La producción pública de medicamentos como parte de una estrategia de política de salud integrada. Reflexiones y recomendaciones para el desarrollo inclusivo en salud. En: THOMAS, Hernán; SANTOS, Guillermo (Coord.). Tecnologías para incluir. Ocho análisis socio-técnicos orientados al diseño estratégico de artefactos y normativas. Buenos Aires: IESCT y Universidad Nacional de Quilmes y Lenguaje Claro, 2016. Agenda CTD. p. 261, 262.

40 SANTOS, Guillermo; BECERRA, Lucas. La producción pública de medicamentos como parte de una estrategia de política de salud integrada. Reflexiones y recomendaciones para el desarrollo inclusivo en salud. En: THOMAS, Hernán; SANTOS, Guillermo (Coord.). Tecnologías para incluir. Ocho análisis socio-técnicos orientados al diseño estratégico de artefactos y normativas. Buenos Aires: IESCT y Universidad Nacional de Quilmes y Lenguaje Claro, 2016. Agenda CTD. p. 263.

41 SANTOS, Guillermo; BECERRA, Lucas. La producción pública de medicamentos como parte de una estrategia de política de salud integrada. Reflexiones y recomendaciones para el desarrollo inclusivo en salud. En: THOMAS, Hernán; SANTOS, Guillermo (Coord.). Tecnologías para incluir. Ocho análisis socio-técnicos orientados al diseño estratégico de artefactos y normativas. Buenos Aires: IESCT y Universidad Nacional de Quilmes y Lenguaje Claro, 2016. Agenda CTD. p. 263, 264.

42 ARGENTINA. Ministerio de Economía y Finanzas Públicas. Secretaría de Política Económica y Planificación del Desarrollo. Subsecretaría de Planificación Económica. Dirección Nacional de Planificación Regional. Dirección Nacional de Planificación Sectorial. Complejo Farmacéutico. Serie Complejos Productivos. 2015. Disponible en internet en: < http://www.economia.gob.ar/peconomica/docs/Complejo_Farmaceutico.pdf>. Consultado el: 7 sept. 2016.

43 ARĞENTINA. Ministerio de Ciencia, Tecnología e Innovación Productiva. Nuevo financiamiento para producción pública de medicamentos tuberculostáticos. 2014. Disponible en internet en: $<$ http://www.mincyt.gob.ar/noticias/nuevo-financiamiento-para-produccion-publica-de-medicamentos-tuberculostaticos-10181>. Consultado el: 7 sept. 2016.

44 ARGENTINA. Ministerio de Ciencia, Tecnología e Innovación Productiva. Nuevo financiamiento para producción pública de medicamentos tuberculostáticos. 2014. Disponible en internet en: $<$ http://www.mincyt.gob.ar/noticias/nuevo-financiamiento-para-produccion-publica-de-medicamentos-tuberculostaticos-10181>. Consultado el: 7 sept. 2016.

\title{
TOWARDS AN INTEGRATED HEALTHPOLICY: COMPULSORY LICENSING AND PUBLIC PRODUCTION OF PHARMACEUTICAL DRUGS.
}

\begin{abstract}
This paper is aimed at describing the mandatory licenses and at presenting the impacts produced by the process of rendering the protection of drugs patents more flexible, so as to recognize the right to undertake alternative measures for public health and to promote the access to pharmaceutical drugs. Beyond that, it introduces the notion of drug production by the public sector as an instrument of public policy and strategic management of health, as a way to reinforce
\end{abstract}


it regarding intellectual property, stimulating research and development, connecting the public and private sectors, ensuring the provision and distribution, thus resulting in the fortification of the dynamics of national innovation. The methods used in the research were comparative and deductive, and the research is bibliographical.

Keywords: Public production of pharmaceutical drugs. Technological innovation. Intellectual property. Pharmaceutical patents. Compulsory licensing.

Submetido: 11 out. 2016

Aprovado: 10 nov. 2016 